\title{
EFECTO DE LA COMPACTACIÓN DEL SUELO EN LA PRODUCCIÓN DE FRIJOL ${ }^{1}$
}

\author{
Claro A. Alfonso 2 , Milagros Monedero ${ }^{2}$, S. Hernandez ${ }^{3}$, Víctor Somoza 2 , Livia Sánchez ${ }^{3}$
}

\begin{abstract}
RESUMEN
Efecto de la compactación del suelo en la producción de frijol. En un suelo Ferralítico Rojo (Oxisol) representativo de La Llanura Roja del sur de La Habana en vías de degradación por compactación, ubicado en la Estación Experimental de Suelos La Renee, se llevó a cabo en el periodo 19931995, un ensayo de simulación de diferentes grados de compactación, obtenidos por medio de pases sucesivos de grada mediana . El objetivo fue definir la relación entre la baja fertilidad que presentan estos suelos y la compactación antrópica. El cultivo indicador fue el frijol, en relación con maíz. La evaluación de la compactación se efectuó mediante perfiles cartográficos, con los cuales se observó y midió: la penetrometría, el estado interno de la estructura y la colonización radical. Los resultados indicaron una relación directa entre la degradación por compactación y la disminución de la porosidad estructural. El espesor y valor de la capa compacta formada aumentó en dependencia del número de pases de grada. De forma similar la colonización radical se vio restringida a la capa suelta superficial, siendo menos favorecida en la variante de 20 pases de grada, donde su profundidad resultó menor. Los rendimientos de frijol y maíz disminuyeron presentando una relación estrecha con la compactación.
\end{abstract}

\begin{abstract}
Effect of the soil compactation on beans production. In a ferralytic red soil (Oxisol) representative of the Red Plains to the South of La Habana, partially degraded by soil density and located in the Soils Experimental Station La Renee, a trial was conducted in 1993-1995, to simulate different degrees of soil density obtained by successive passes of tillage. The objective was to determine the relationship between the low fertility of these soils and the man-induced soil density. The indicator crop used was the common bean in succession with maize. The evaluation of soil density was carried out through cartographic profiles, which were used to observe and measure: the degree of penetration; the state of the internal structure, and root colonization. Results indicate a direct correspondence between degradation by soil density and a reduction in structural porosity. Similarly, the thickness of the compacted layer increased depending on the number of passes of tillage, and root colonization that was restricted to the loose surface layer, being least favorable in the treatment of 20 passes, in which its depth was the less. Yields of beans and maize presented a close relationship with soil density, being reduced as density increased. Therefore, the maninduced soil density that results from intensive cultivation of these soils is the cause of low fertility.
\end{abstract}

\section{INTRODUCCIÓN}

La existencia de un horizonte o de un sub-horizonte compacto, sea en la superficie, en el horizonte Ap o en profundidad, puede tener como origen causas naturales, o resultar de la acción del hombre, por ejemplo, la formación de una suela o piso de labor. Estos horizontes constituyen una preocupación permanente para los pedólogos, microbiólogos, agrónomos y agricultores del mundo entero, porque engendran directa o indi- rectamente fenómenos tales como: pérdidas de suelos por erosión superficial, arrastre que conlleva pérdidas de elementos nutritivos, disminución de la porosidad y destrucción de la estructura. También baja de la actividad biológica, un obstáculo al desarrollo radical, y finalmente, una caída de los rendimientos. El conjunto de estos fenómenos y en consecuencia la aparición de un horizonte compacto y los daños que produce son considerados como una degradación del suelo (Alfonso 1987).

\footnotetext{
1 Presentado en la XLV Reunión Anual del PCCMCA, Guatemala 1999.

2 Estación Experimental La Renee del Instituto de suelos del MINAG. Km 33 1/2 carretera de Bejucal a Quivicán. Habana Cuba. E-mail: larenee@ceniai.inf.cu

3 Estación Experimental de San Juan y Martínez, Finca Obeso, Pinar del Río.
} 
Los suelos de la Llanura Roja al sur de la Provincia de La Habana, son uno de los agroecosistemas más intensamente cultivados de Cuba. El ritmo de laboreo es muy elevado, sin tener en cuenta en muchos casos el grado de humedad con que se trabaja el suelo, pudiendo llegar en total en un año alrededor de 30 labores, sin considerar el efecto degradante de los sistemas de riego (DDA-100M, Pivote Central, Bochankas) cuando los cultivos aún no han cubierto la superficie. Por último, los vehículos que trafican durante la cosecha también producen compactación. Se presenta entonces un calendario de actividades muy sobrecargado, que ha contribuido a través de los años a la pérdida de la fertilidad de los suelos.

Por todo ello el objetivo del presente ensayo fue estudiar las modificaciones sufridas por la fertilidad del suelo después de una compactación forzada por el hombre (particularmente las consecuencias sobre el estado interno de la estructura), la colonización radical y la evolución de los rendimientos en relación con el desarrollo de la capa compacta.

\section{MATERIALES Y MÉTODOS}

En un suelo Ferralítico Rojo (Oxisol) ubicado en La Estación Experimental de Suelos La Reneé, del Municipio de Quivicán, al sur de la Provincia de La Habana, se llevó a cabo un ensayo de simulación de diferentes grados de compactación, mediante $(0,5,10,15 \mathrm{y}$ 20) pases sucesivos de grada mediana (2500 lbs). El experimento se inició en la campaña de frío de 1993-1994 y se le dio seguimiento en la de 1994-1995. La rotación seguida fue: frijol - maíz - frijol - maíz. Los bloques de preparación fueron de una superficie de $2000 \mathrm{~m}^{2}$.
La rotura y cruce se realizó con arado de discos hasta $25-30 \mathrm{~cm}$ de profundidad. La humedad cuando se pasó la grada fue de alrededor de $80-85 \%$ de la capacidad de campo. Para evaluar la compactación producida se excavaron perfiles en el período R-7 del frijol, en los que se determinó la penetrometría (con penetrómetro de bolsillo), se observó el estado interno de la estructura por el método cartográfico de Manichon (1982-a) y la colonización radical según el método cartográfico de Tardieu (1984) modificado por Alfonso (1987). Paralelamente se determinó la velocidad de filtración con infiltrómetro de laboratorio, la densidad aparente con cilindros y la porosidad interagregados (porosidad estructural) tanto del lecho superior como de la capa compacta. Las variedades de frijol y maíz utilizadas fueron BAT-304 y T- 66. Las distancias de siembra fueron: $0,70 \mathrm{~m} \times 0,05 \mathrm{~m}$ para el frijol y $0,90 \mathrm{~m} \times 0,30 \mathrm{~m}$ para el maíz. El cultivo se desarrolló bajo riego por aspersión. El rendimiento fue determinado mediante el muestreo de cuatro subparcelas de $35 \mathrm{~m}^{2}$ por cada tratamiento (bloque de $2000 \mathrm{~m}^{2}$ ) distribuidas al azar. Sus valores fueron evaluados mediante un esquema de Bloques al Azar y sus medias comparadas según la Prueba de Rango Múltiple de Duncan.

\section{RESULTADOS Y DISCUSIÓN}

El incremento de las labores con grada produjo deterioro físico de la estructura, manifestándose en una compactación de los componentes elementales que forman la estructura al nivel subsuperficial. En el Cuadro 1 se observan las modificaciones de algunas variables físicas analizadas. La porosidad estructural o interagregados se redujo progresivamente en la zona susceptible a compactarse y varió de $25 \%$ en el testigo hasta $12 \%$

Cuadro 1. Modificaciones de algunas propiedades físicas causadas por la compactación.

\begin{tabular}{|c|c|c|c|c|c|c|c|c|}
\hline \multirow[t]{2}{*}{$\begin{array}{c}\text { No de pases de } \\
\text { de grada }\end{array}$} & \multirow[t]{2}{*}{ Capa } & \multicolumn{3}{|c|}{ Porosidad $(\%) *$} & \multicolumn{2}{|c|}{$\begin{array}{l}\text { Velocidad de filtración } \\
\text { piso de labor }(\mathrm{mm} / \mathrm{h})\end{array}$} & \multicolumn{2}{|c|}{$\begin{array}{l}\text { Densidad } \\
\left(\mathrm{g} / \mathrm{cm}^{3}\right)\end{array}$} \\
\hline & & P.G. & P.T. & P.E. & Surco & Calle & Surco & Calle \\
\hline \multirow[t]{2}{*}{1} & Suelta & 67 & 40 & 27 & - & - & 1,02 & 1,08 \\
\hline & $>20 \mathrm{~cm}$ & 65 & 40 & 25 & 325 & 215 & 1,05 & 1,12 \\
\hline \multirow[t]{2}{*}{5} & Suelta & 66 & 40 & 26 & - & - & 1,03 & 1,10 \\
\hline & Compacta & 64 & 46 & 18 & 125 & 98 & 1,16 & 1,22 \\
\hline \multirow[t]{2}{*}{10} & Suelta & 65 & 39 & 26 & - & - & 1,03 & 1,10 \\
\hline & Compacta & 63 & 47 & 16 & 85 & 53 & 1,18 & 1,25 \\
\hline \multirow[t]{2}{*}{15} & Suelta & 65 & 40 & 25 & - & - & 1,03 & 1,10 \\
\hline & Compacta & 60 & 47 & 13 & 70 & 45 & 1,25 & 1,32 \\
\hline \multirow[t]{2}{*}{20} & Suelta & 65 & 40 & 25 & - & - & 1,06 & 1,12 \\
\hline & Compacta & 60 & 48 & 12 & 62 & 40 & 1,34 & 1,45 \\
\hline
\end{tabular}

*P.G. = Porosidad Global

P.T. $=$ Porosidad Textural

P.E. $=$ Porosidad Estructural $(\mathrm{PE}=\mathrm{PG}-\mathrm{PT})$ 
como valor extremo en el tratamiento de 20 pases de gradas. Este comportamiento coincide con el descrito por Alfonso et al (1995 y 1997) para condiciones de cultivo intensivo de los suelos Ferralíticos Rojos del Sur de La Habana. Por su parte, Porta et al. (1994) plantean que la intensidad del laboreo está íntimamente relacionada con la tasa de mineralización de la materia orgánica, la que aumenta al roturar un terreno y someterlo a un laboreo intenso y continuo. Ello hace más vulnerable la estructura del suelo al disminuir su estabilidad como consecuencia de la caída del tenor de materia orgánica, lo cual facilita la compactación. Los valores de penetrometría atribuibles a los diferentes tratamientos de compactación, permitieron establecer una relación directa con la porosidad estructural (Figura 1), lo que muestra que a partir del tratamiento con cinco pases de grada, se presentaron las diferentes gradaciones de la compactación. Estos resultados concuerdan con las mediciones realizadas al respecto por Sánchez et al (1994) en ensayos con diferentes sistemas de labranza. La capa suelta superior del perfil con una estructura fragmentaria suelta (tipo $F \Gamma$ ) según Manichon (1984-a), no presentó variaciones significativas en sus propiedades físicas. Sin embargo su espesor o profundidad disminuyó en función de los tratamientos, de forma tal que en la variante extrema, este lecho presentó un espesor mucho menor que en los demás tratamientos, en relación con el espesor o volumen útil de los suelos para la planta. Para Altieri (1994) existe una relación estrecha entre el Uso Máximo Sustentable (MSU) del suelo agrícola y la profundidad del suelo, lo cual afecta la tasa de renovación de la fertilidad.

Este comportamiento con respecto a la capa superior por el contrario y de forma inversamente propor- cional, redunda en un aumento del espesor y de la compactación (tipo M $\Delta$ según Manichon 1984-a). Este comportamiento se nota mucho mejor al vincular esta capa con la colonización radical del frijol en función de los tratamientos (Figuras 2a, 2b y 2c). Esto corrobora que el volumen de suelo a colonizar por éstas disminuyó en función del espesor y grado de compactación, siendo el valor de $3,25 \mathrm{~kg} / \mathrm{cm}^{2}$ el umbral crítico $(10$ pases de grada), para la colonización radical y los tratamientos de 10,15 y 20 pases de grada, donde las raíces se deformaron al atravesarlas, Alfonso et al. (1995). Obtuvo un comportamiento similar en condiciones controladas con el cultivo del frijol. Por último, los rendimientos en grano obtenidos por el frijol y el maíz, (Cuadro 2), se vieron afectados conforme al grado de compactación producida por los tratamientos aumentó con relación al testigo, siendo significativos sus valo-

Cuadro 2. Efecto de la compactación sobre los rendimientos de maíz y frijol.

\begin{tabular}{|c|c|c|c|c|}
\hline \multirow{3}{*}{$\begin{array}{l}\text { No de pases } \\
\text { de grada }\end{array}$} & \multicolumn{4}{|c|}{ Rendimientos (t/ha) } \\
\hline & \multicolumn{2}{|c|}{$1993-1994$} & \multicolumn{2}{|c|}{$1994-1995$} \\
\hline & Frijol & Maíz & Frijol & Maíz \\
\hline 1 & $1,40^{\mathrm{a}}$ & $1,75^{\mathrm{a}}$ & $1,10^{\mathrm{a}}$ & $1,54^{\mathrm{a}}$ \\
\hline 5 & $1,29^{\mathrm{ab}}$ & $1,60^{b}$ & $1,08^{\mathrm{a}}$ & $1,48^{\mathrm{a}}$ \\
\hline 10 & $1,22^{b}$ & $1,42^{\mathrm{c}}$ & $0,96^{b}$ & $1,20^{\mathrm{b}}$ \\
\hline 15 & $1,10^{\mathrm{c}}$ & $1,05^{\mathrm{d}}$ & $0,90^{\mathrm{b}}$ & $1,00^{\mathrm{c}}$ \\
\hline 20 & $1,08^{\mathrm{c}}$ & $0,95^{\mathrm{d}}$ & $0,83^{\mathrm{c}}$ & $0,83^{\mathrm{d}}$ \\
\hline EsX & $0,007 * *$ & $0,012 * *$ & $0,009 * *$ & $0.017 * *$ \\
\hline
\end{tabular}

$* * \mathrm{P}<0,01$

a,b,c,d, letras en común no difieren significativamente, según Prueba de Rango Múltiple de Duncan a $\mathrm{P}<5 \%$.

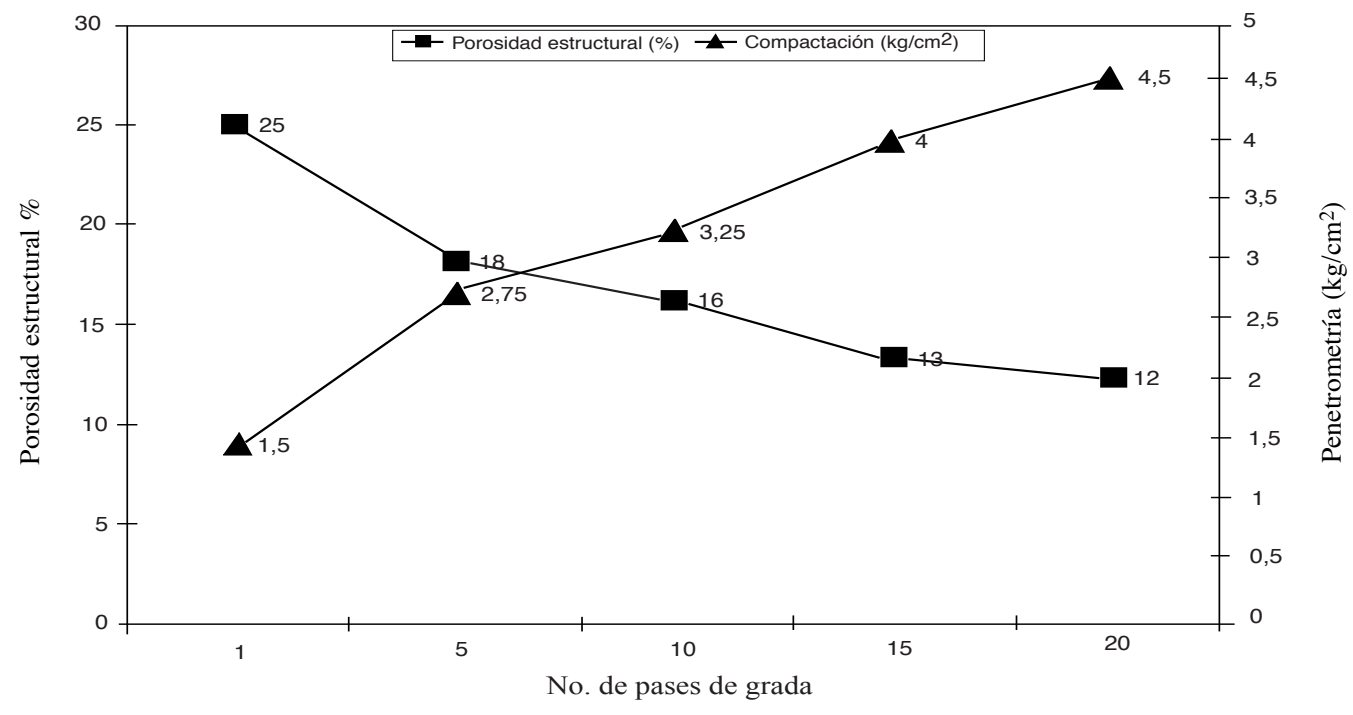

Figura1. Impacto del laboreo con grada sobre la compactación y la porosidad estructural (\%). 


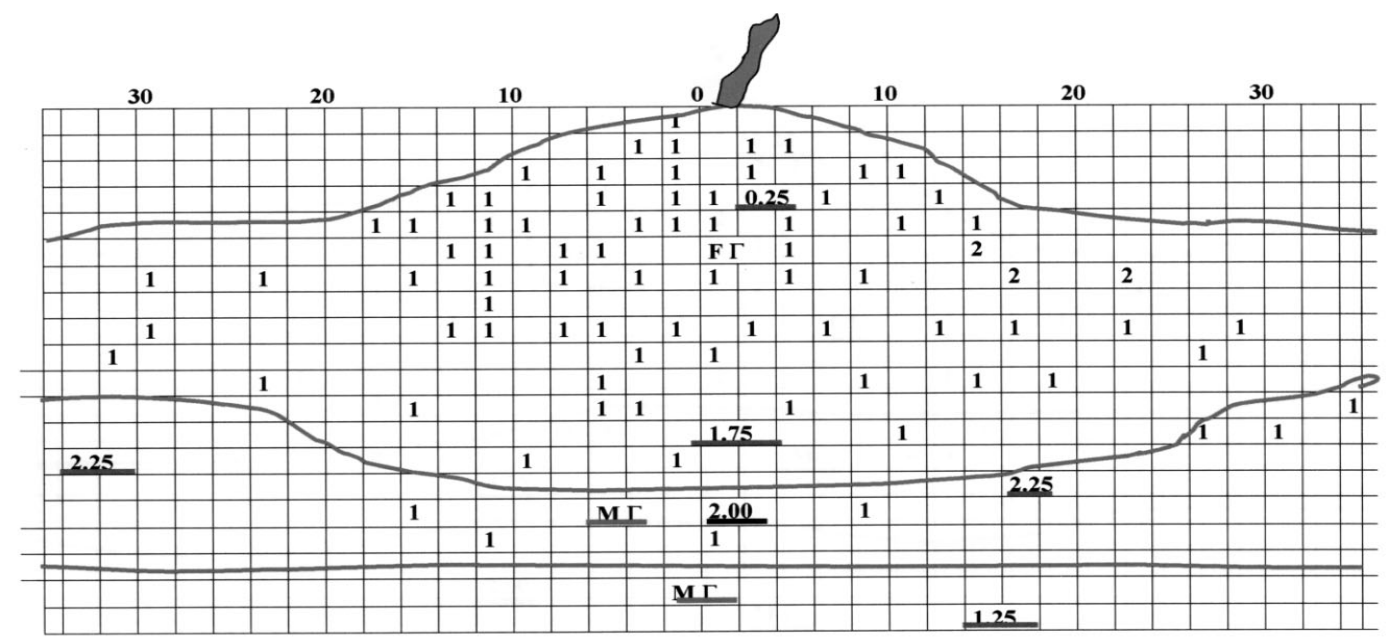

Figura 2.a. Perfil radical vertical del tratamiento sin pases de grada.

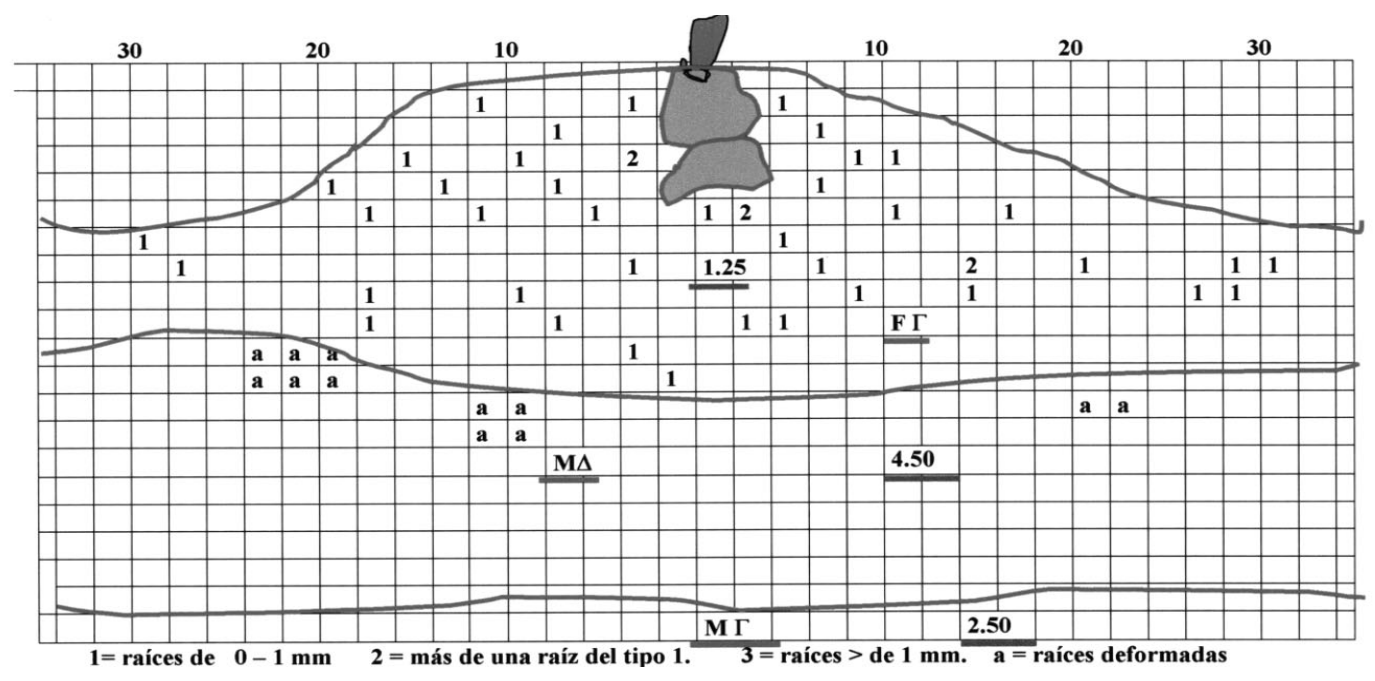

Figura 2.b. Perfil radical vertical del tratamiento con 10 pases de grada.

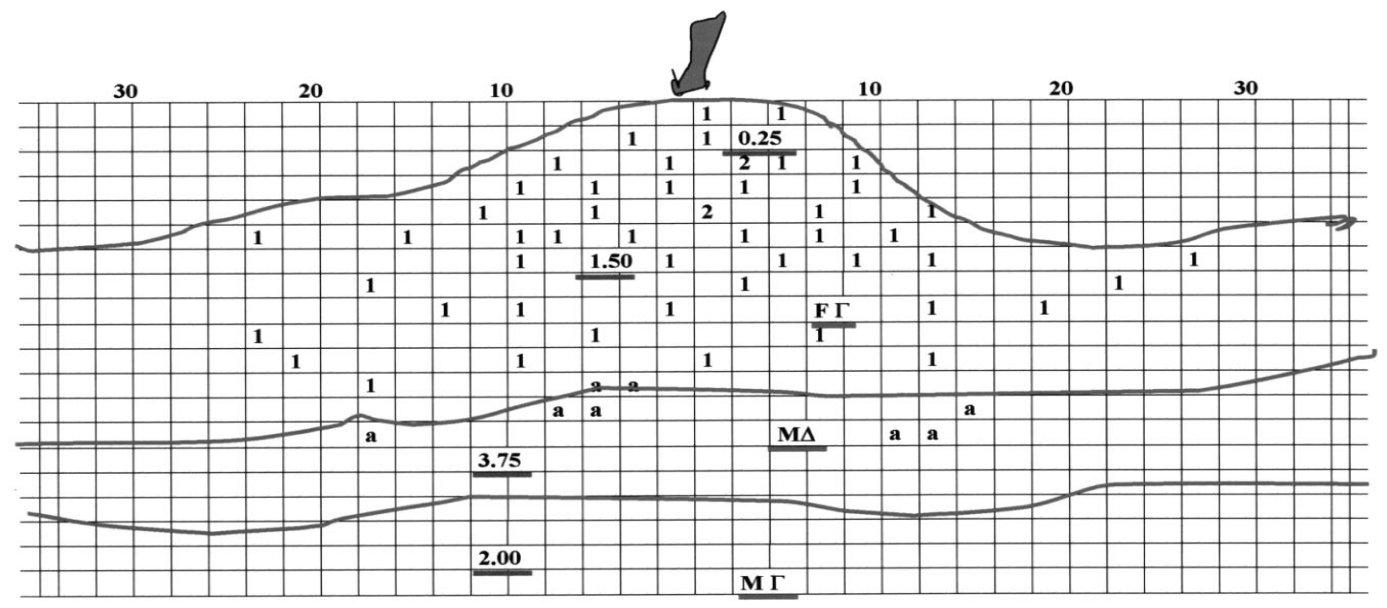

Figura 2.c. Perfil radical vertical del tratamiento con 20 pases de grada. 
res a partir del tratamiento de 10 pases de grada. Esta respuesta de la planta puede relacionarse con la disponibilidad de los nutrimentos al reducirse el volumen de prospección de las raíces al desaparecer o reducirse al mínimo la porosidad estructural. En consecuencia, la fertilidad de ese suelo se hace insostenible, al no existir una disponibilidad permanente de nutrimento. (Porta et al., 1994).

\section{CONCLUSIONES}

Existió una marcada influencia negativa de los grados de compactación provocados por los tratamientos de laboreo intensivo, comparados con el testigo, con respecto a la porosidad estructural, la colonización radical y los rendimientos de frijol y maíz.

En consecuencia, la fertilidad física se vio afectada negativamente en función de la compactación antrópica producto del cultivo intensivo de este suelo, lo cual es causa de su baja fertilidad.

\section{LITERATURA CITADA}

ALFONSO, C.A.; MOREJON, L.; La O, M.; NAPOLES, M.; GARCIA, M.E. 1997. Recuperación de áreas con problemas de compactación dedicadas al cultivo de la papa del sur de La Habana. Ponencia al XI FORUM de Ciencia y Técnica. 13p.

ALFONSO, C.A. 1995. Determinar las tecnologías de preparación y acondicionamiento más adecuadas de los suelos, incluyendo fertilización y uso de abonos verdes en la rotación de los cultivos. Informe Final Resultado $00502.35 \mathrm{p}$.

ALFONSO, C.A.; HERNANDEZ, J.G.; DREVON, J.J. 1995. Influencia de la compactación en el desarrollo radical del frijol en condiciones controladas. Ponencia presentada al Congreso Latinoamericano de Rhizobiología. La Habana. 9 p.

ALFONSO, C.A. 1987. Etude de la degradation des sols cultives en tabac (Cuba, province de Pinar del Río). These soutenue pour le titre de Docteur Ingénieur. INA -PG. France. 188 p.

ALTIERI, M.A.; 1994. Bases agroecológicas para una producción agrícola sustentable. Agricultura Técnica (Chile) 54 (4): 371-386.

MANICHON, H. 1982 b. Influence des systemes de culture sur le profil cultural: élaboration d'une méthode de diagnostic basée sur l'observation morphologique. These soutenue pour le titre de Docteur Ingénieur. INA - PG. France. $214 \mathrm{p}$.

RIVEROL, M.; ALFONSO, C.A. 1995. La degradación de los suelos. Alternativas para su mejoramiento. II Encuentro Nacional de Agricultura Orgánica. ICA. Cuba. pp. $16-19$.

PORTA,J.; LOPEZ, M.; ROQUERO, C. 1994. Edafología para la Agricultura y el Medio Ambiente. Ed. Mundi Prensa. Madrid. 625 p.

SANCHEZ, L.; HERNADEZ, S.; ALFONSO,C.A.; URIARTE, R. 1994. Evaluación de diferentes métodos de manejo a través de la resistencia del suelo a la penetración. Memorias XXV Aniversario del Instituto de Suelos. $8 \mathrm{p}$. 